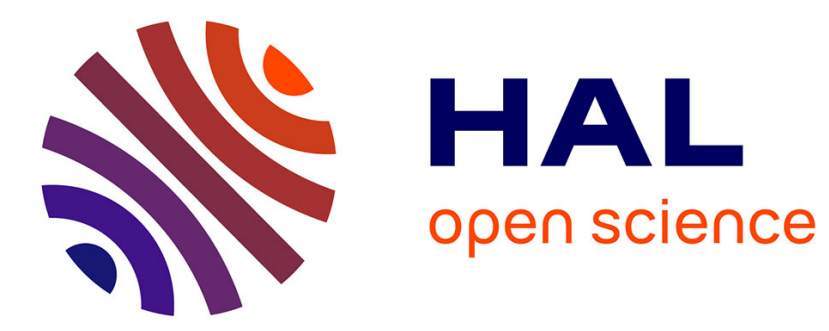

\title{
Réalisation et expérimentation d'une génératrice unipolaire impul sionnelle sans fer de très grande puissance massique
}

\author{
R. Hahn, J. Lucidarme, C. Rioux, R. Guillet
}

\section{To cite this version:}

R. Hahn, J. Lucidarme, C. Rioux, R. Guillet. Réalisation et expérimentation d'une génératrice unipolaire impul sionnelle sans fer de très grande puissance massique. Revue de Physique Appliquée, 1973, 8 (1), pp.53-66. 10.1051/rphysap:019730080105300 . jpa-00243654

\section{HAL Id: jpa-00243654 https://hal.science/jpa-00243654}

Submitted on 1 Jan 1973

HAL is a multi-disciplinary open access archive for the deposit and dissemination of scientific research documents, whether they are published or not. The documents may come from teaching and research institutions in France or abroad, or from public or private research centers.
L'archive ouverte pluridisciplinaire HAL, est destinée au dépôt et à la diffusion de documents scientifiques de niveau recherche, publiés ou non, émanant des établissements d'enseignement et de recherche français ou étrangers, des laboratoires publics ou privés. 


\title{
RÉALISATION ET EXPÉRIMENTATION \\ D'UNE GÉNÉRATRICE UNIPOLAIRE IMPULSIONNELLE SANS FER DE TRÈS GRANDE PUISSANCE MASSIQUE
}

\author{
R. HAHN, J. LUCIDARME, C. RIOUX et R. GUTLLET \\ Laboratoire de Physique des Plasmas, Groupe Electrotechnique et Fusion Contrôlée \\ Université Paris-Sud, Centre d’Orsay
}

(Reçu le 5 septembre 1972)

\begin{abstract}
Résumé. - La recherche de la production de hautes puissances électriques nécessaires à la réalisation d'expériences de physique des plasmas dans le domaine des fortes densités nous a conduit à l'étude et à la réalisation de machines électrotechniques spéciales.

Cet article, après avoir rappelé les motivations constructives, décrit l'étude théorique et expérimentale de la dynamo unipolaire impulsionnelle de 5 MJ.

L'expérimentation systématique a montré la validité des équations d'évolution obtenues dans l'étude théorique, et a mis en évidence le comportement des balais liquides en mercure dans la commutation des fortes intensités mises en jeu. Nous décrivons les instabilités observées des balais liquides et nous donnons un critère de stabilité.
\end{abstract}

\begin{abstract}
We have constructed and experimented with a special electric machine coupled to a high moment of inertia fly-wheel accumulating a kinetic energy of $5 \mathrm{MJ}$ in order to generate high electric impulse power. The generator is a self-excited, ironless, homopolar dynamo. Current collection has been achieved by the use of a liquid metal brush system.

This report describes the overall generator fly-wheel system, as well as the obtained experimental results.
\end{abstract}

1. Introduction. - La recherche de la production de hautes puissances électriques nécessaires à la réalisation d'expériences de physique des plasmas dans le domaine des fortes densités nous a conduit à l'étude et à la réalisation de machines électrotechniques spéciales.

Une étude critique de l'électrotechnique [1] a montré que la puissance maximale possible d'une machine électrique est proportionnelle au carré du champ d'induction. Dans les machines classiques, ce dernier est limité aux environs de 1,5 T. Pour obtenir des performances supérieures, l'utilisation de champs d'induction plus élevés, par exemple de 5 ou 6 T s'impose. Cette dernière condition implique l'abandon des matériaux ferro-magnétiques qui deviennent inutiles, on aboutit ainsi à la conception de machine «sans fer ».

La filière développée au Laboratoire de Physique des Plasmas d'Orsay se base sur l'exploitation constructive des considérations générales que nous venons de donner. Bien évidemment, la suppression du fer entraîne de profonds changements dans l'architecture des machines, et provoque l'apparition de problèmes nouveaux. En effet, par suite de la présence du fer, la création du champ d'excitation d'une machine classique ne demande qu'une faible puissance. Dans une machine sans fer, au contraire, le champ d'excitation n'est obtenu qu'au prix de courants très intenses, dissipant des puissances Joule se chiffrant en mégawatts. Cette difficulté a été levée en choisissant un montage autoexcité. Dans ce cas, la génératrice fournit elle-même la puissance d'excitation qui lui est nécessaire.

Enfin, la haute puissance massique de ces machines, indispensable pour obtenir une délivrance rapide de l'énergie, pose de nombreux problèmes de résistance des matériaux. Pour les résoudre, une structure compacte unipolaire a été adoptée. Elle se prête particulièrement bien par sa géométrie entièrement de révolution, à des sollicitations mécaniques intenses.

Sur ces principes une dynamo unipolaire impulsionnelle autoexcitée de $5 \mathrm{MJ}$ a été réalisée au laboratoire. Elle a atteint le stade opérationnel dès 1968. Nous allons la décrire, exposer son principe de fonctionnement, et enfin nous donnerons les principaux résultats expérimentaux.

La conception de cette génératrice a bénéficié de l'expérience acquise lors de la réalisation et de l'expérimentation d'une génératrice impulsionnelle de $30 \mathrm{~kJ}$ qui a fonctionné dans notre laboratoire en 1961 [2].

1.1 DESCRIPTION DE LA GÉNÉRATRICE. - Le principe de fonctionnement de la dynamo unipolaire impul- 
sionnelle consiste à transformer brutalement l'importante énergie cinétique emmagasinée dans un volant de grand moment d'inertie en rotation rapide en énergie électrique. Celle-ci est délivrée sous la forme d'une impulsion de courant de haute intensité pendant le temps de freinage du volant. La commutation de ces hautes intensités mises en jeu est assurée par des balais liquides en mercure.

La figure 1 représente schématiquement la dynamo impulsionnelle. Elle est constituée essentiellement d'un ensemble volant-rotor et d'un stator, tous deux de révolution autour de l'axe de rotation du rotor.

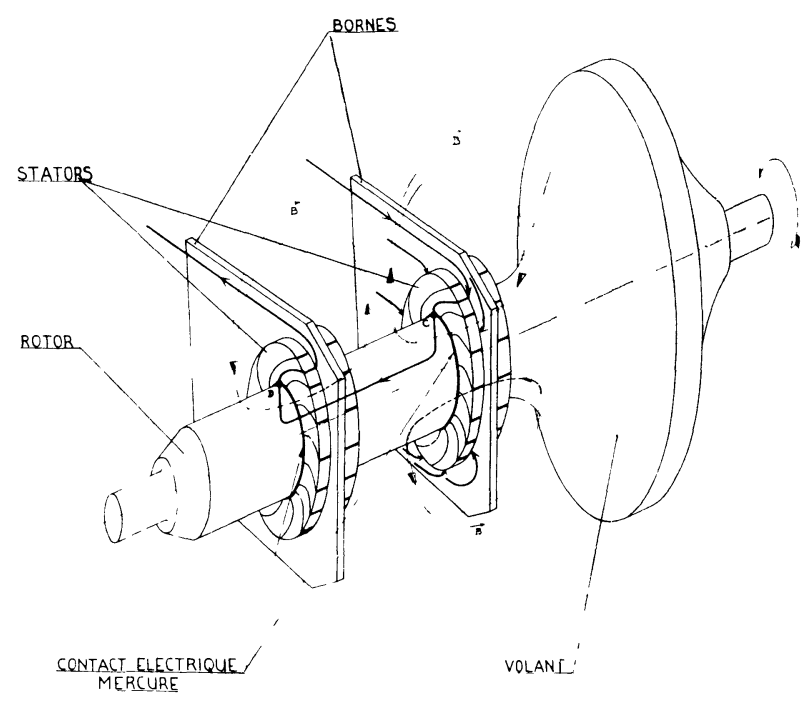

FIG. 1.

L'ensemble volant-rotor a été réalisé d'un seul bloc en bronze au bérylium. Ce matériau, non magnétique, a été choisi pour ses qualités électriques et mécaniques. En effet, lors du fonctionnement de la dynamo, le rotor est parcouru par un courant élevé et il subit des contraintes mécaniques importantes dues à la grande vitesse de rotation et au couple atteignant plusieurs mètres-tonnes transmis du volant à la partie électrique du rotor.

Le stator en cuivre massif, est constitué de deux blocs symétriques. Des rainures en forme de spirale sont percées dans la masse du stator et sont comblées d'isolant. Le courant de la machine, passant dans les feuillets de cuivre ainsi formés, possède une composante azimutale et crée le champ magnétique $B$ dont les lignes de force sont représentées sur la figure 1. Une bague de court-circuit collecte le courant du feuilletage pour le distribuer aux contacts liquides.

La rotation du rotor dans le champ magnétique $B$ induit entre les points $C$ et $D$ une force électromotrice proportionnelle à la vitesse du rotor et au courant de la génératrice. Celle-ci est ainsi autoexcitée.

L'injection de mercure sous forte pression entre le rotor et les deux demi-stators réalise les contacts électriques tournants et ferme le circuit électrique interne à la génératrice. Cette injection de mercure est effectuée à l'aide de deux vérins, isolés électriquement, et alimentant chacun un contact tournant.

1.2 Mise EN ceuvre. - La mise en œuvre de la génératrice s'effectue en quatre opérations successives. Tout d'abord, le volant est lancé progressivement par un moteur de faible puissance, dernier élément d'un groupe Ward-Léonard. $\mathrm{Ce}$ système permet d'obtenir un couple important dans un grand domaine de vitesse. Lorsque la vitesse de fonctionnement est atteinte, le volant, entraîné par l'intermédiaire d'un embrayage électromagnétique, est désaccouplé du moteur d'entraînement. Ensuite, deux bobines annexes d'excitation, placées entre les deux demi-stators créent un faible champ magnétique initial nécessaire à l'amorçage de la dynamo. Enfin, l'injection du mercure sous pression dans les intervalles rotor-stator ferme le circuit électrique de la génératrice. Une impulsion de courant se développe alors par autoexcitation, tandis que le rotor est violemment freiné par le couple électromagnétique. Ainsi une partie de l'énergie cinétique accumulée dans le volant est transférée sous forme d'énergie électrique à la charge de la dynamo.

1.3. DESCRIPTION DU CIRCUIT MERCURE. - La propriété que possède le mercure de mouiller les surfaces de cuivre a pour conséquence de réduire fortement les résistances électriques de contact. Il est ainsi possible de commuter par des balais liquides en mercure des intensités élevées, de l'ordre de $10000 \mathrm{~A} \mathrm{par} \mathrm{cm}^{2}$, avec des chutes ohmiques négligeables. Notons cependant que la chaleur dégagée par effet Joule est telle qu'il est nécessaire de faire circuler le mercure.

Nous allons décrire plus précisément le circuit d'injection du mercure et les balais liquides, étant donné leur rôle important dans le fonctionnement de la génératrice.

1.3.1 Description du système d'injection du mercure. - Une représentation schématique du système d'injection est donnée figure 2. Ce dispositif a

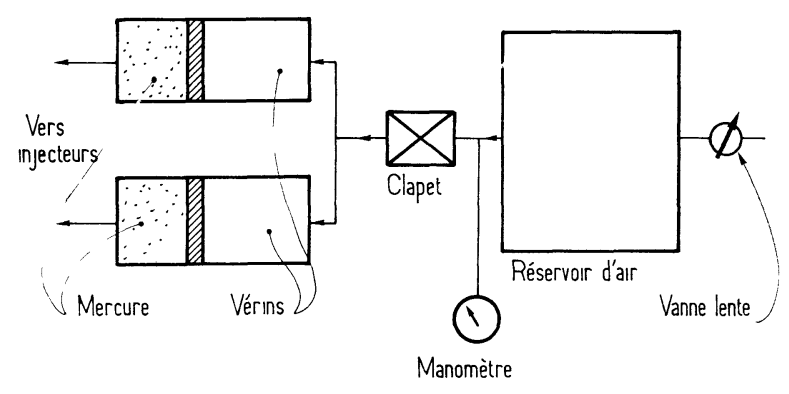

FIG. 2.

pour but d'injecter un grand volume de mercure sous une pression élevée dans les intervalles rotor-stator, afin d'y assurer un excellent contact électrique et une circulation rapide $\mathrm{du}$ mercure. 
Il comprend :

- Un compresseur d'air.

- Un réservoir d'air comprimé constitué de deux bouteilles de 2001 pouvant être gonflées jusqu'à une pression de 200 bars : il est destiné à maintenir pendant le temps de fonctionnement une pression sensiblement constante.

- Une soupape à ouverture rapide commandée par un électro-aimant.

- Deux vérins d'injection d'une contenance maximum de 91 chacun. Dans ces vérins le mercure est poussé à l'aide d'air comprimé par l'intermédiaire d'un piston libre, dont la position initiale réglable détermine le volume de mercure injecté. Chaque vérin, isolé électriquement, alimente un contact mercure par deux tubes de forte section.

1.3.2 Description du circuit interne à la génératrice. - Le circuit mercure interne à la machine est représenté sur la figure 3.

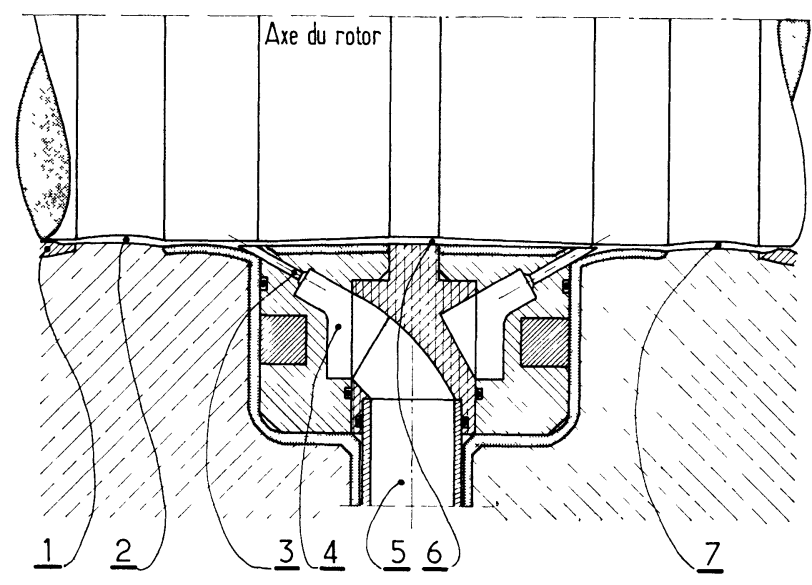

FIG. 3.

Les deux contacts électriques 2 et 7 et leur alimentation en mercure sont identiques. Les deux tubes 5 provenant d'un vérin débouchent dans une chambre annulaire 4 d'où partent 56 trous de $4 \mathrm{~mm}$ de diamètre 3 et qui injectent axialement le mercure dans l'intervalle rotor-stator. Ensuite l'écoulement a lieu entre deux cylindres coaxiaux jusqu'à la zone de contact électrique. La surface intérieure du stator, recouverte d'une couche d'isolant, forme le cylindre extérieur ; le rotor, non isolé, forme le cylindre intérieur. Après un séjour dans l'intervalle 2 le mercure est évacué vers l'extérieur au travers de l'étranglement 1 . Une partie du mercure s'introduit durant la détente dans l'intervalle 6 .

Afin de rendre négligeable les pertes de charge, la section droite de l'ensemble des tuyaux d'arrivée est supérieure à la section droite totale des étranglements.

Les dimensions des contacts électriques ont été déterminées en fonction de la courbure des lignes de force du champ magnétique, de la résistance élec- trique des contacts et du jeu mécanique nécessaire au montage. Ceci nous a conduit à adopter les dimensions suivantes :

- longueur du contact $30 \mathrm{~mm}$,

- épaisseur du contact $1,5 \mathrm{~mm}$.

Le diamètre du rotor étant $2 r=154 \mathrm{~mm}$. Un étranglement de $0,6 \mathrm{~mm}$ doit assurer, d'une part le remplissage correct du contact en conservant la pression en amont de l'écoulement, d'autre part, une vitesse de circulation du fluide suffisante pour éviter un échauffement trop important.

2. Étude théorique de la dynamo unipolaire. Nous allons étudier un premier modèle simplifié de la dynamo unipolaire pour lequel nous supposerons constants les principaux paramètres électriques (self, résistance interne, coefficient de couplage électromécanique rotor-stator). A l'aide de ce modèle, nous dégagerons les principales propriétés de cette machine.

2.1 ÉVOLUTION DU SYSTÈME SANS FROTTEMENT MÉCANIQUE ET SANS EFFET D'ÉCRAN ÉLECTROMAGRÉTIQUE. L'injection du mercure dans l'intervalle stator-rotor est accompagnée de pertes mécaniqu es dues à la viscosité du fluide. D'autre part, les courants induits dans les diverses pièces métalliques de révolution exercent sur le flux principal des effets d'écran tendant à étouffer celui-ci. Nous négligeons tout d'abord ces perturbations; nous modifierons ensuite les équations pour en tenir compte.

2.1.1 Équations d'évolution. - Considérons les grandeurs instantanées :

$\Phi$ le flux magnétique, orienté de l'intérieur vers l'extérieur, au travers de la surface cylindrique comprise entre les balais.

$j$ l'intensité du courant.

$\omega$ la vitesse angulaire de rotation du rotor.

Le flux $\Phi$ créé par la composante azimutale du courant $j$ circulant dans les stators est proportionnel à $j$.

Nous posons

$$
\Phi=2 \pi \alpha j
$$

et nous appellerons la constante $\alpha$ coefficient de couplage électromécanique puisqu'elle exprime le couplage rotor-stator.

La force électromotrice $u$ engendrée entre les balais est :

$$
u=\frac{\Phi}{2 \pi} \cdot \omega=\alpha \omega j
$$

Le couple électromagnétique est lié à la puissance électrique par la relation

$$
C \omega+u j=0
$$

d'où :

$$
C=-\alpha j^{2} .
$$


Nous appelons : $R, L$, la résistance totale et la self totale du circuit électrique de la génératrice fermée sur une charge extérieure et $I$ le moment d'inertie de l'ensemble rotor-volant.

Les équations d'évolution électrique et mécanique peuvent s'écrire :

$$
\begin{aligned}
\alpha \omega j & =L \frac{\mathrm{d} j}{\mathrm{~d} t}+R j \\
I \frac{\mathrm{d} \omega}{\mathrm{d} t} & =-\alpha j^{2} .
\end{aligned}
$$

Nous appelons vitesse critique le rapport

$$
\omega_{c}=\frac{R}{\alpha} \text {. }
$$

L'éq. (1) écrite sous la forme

$$
\frac{1}{j} \frac{\mathrm{d} j}{\mathrm{~d} t}=\frac{\alpha}{L}\left(\omega-\omega_{c}\right)
$$

montre que $\mathrm{d} j / \mathrm{d} t$ est du signe de $\left(\omega-\omega_{c}\right)$. Par conséquent, pour que le courant initial créé par les bobines d'excitation annexes soit amplifié, il faut que la vitesse algébrique initiale $\omega_{m}$ soit supérieure à une constante $\omega_{c}$.

L'éq. (2) montre que $\mathrm{d} \omega / \mathrm{d} t$ est toujours négatif ; la vitesse est toujours décroissante.

2.1.2 Intégrale première. Représentation de l'évo. lution. - Le temps $t$ ne figure pas explicitement dans les équations. Eliminons le temps entre les éq. (1) et (2), nous obtenons :

$$
L j \mathrm{~d} j+I\left(\omega-\omega_{c}\right) \mathrm{d} \omega=0 .
$$

Une intégrale première de l'évolution du système est :

$$
\frac{1}{2} L j^{2}+\frac{1}{2} I\left(\omega-\omega_{c}\right)^{2}=E_{0} .
$$

$E_{0}$ est une constante déterminée par les conditions suivantes :

- A l'instant initial, le courant correspondant au flux initial créé par les deux bobines d'excitation annexes étant négligeable devant le courant délivré par la machine nous avons : $j \# 0, \omega=\omega_{m}$ d'où

$$
E_{0}=\frac{1}{2} I\left(\omega_{m}-\omega_{c}\right)^{2} \text {. }
$$

- A l'instant où $j=j_{\text {max }}, \omega=\omega_{c}$ d'où

$$
E_{0}=\frac{1}{2} L j_{\max }^{2} \text {. }
$$

$E_{0}$ représente donc l'énergie cinétique qui sera transformée, au cours de l'impulsion en énergie électromagnétique.

Dans un diagramme en $\sqrt{\frac{L}{I}} j, \omega$ (Fig. 4), la courbe représentative de l'évolution du système est un cercle de centre $\left(j=0, \omega=\omega_{c}\right)$ de rayon

$$
\omega_{m}-\omega_{c}=\sqrt{\frac{L}{I}} j_{\max } .
$$

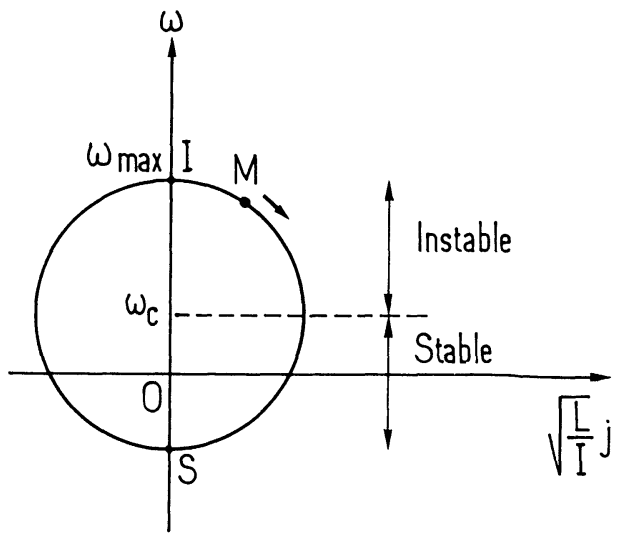

FIG. 4.

Le point $M$ représentatif de l'état du système décrit au cours du temps le cercle (c) dans le sens des $\omega$ décroissants $(\mathrm{d} \omega / \mathrm{d} t$ est toujours négatif). Le fonctionnement normal de la génératrice consiste à passer d'une position initiale $I$ instable, à une position finale $S$ stable. Au cours de cette transition un courant intense se développe, et la vitesse chute brusquement, celle-ci peut s'inverser si la vitesse initiale est suffisamment grande $\left(\omega_{m}>2 \omega_{c}\right)$.

Le sens du courant est déterminé par le sens de l'excitation initiale et reste le même durant l'impulsion.

2.1.3 Expressions explicitées de l'évolution temporelle. - En éliminant le terme $\left(\omega-\omega_{c}\right)$ entre l'intégrale première (3) et les éq. (1) et (2), nous obtenons après intégration les expressions de $j(t)$ et $\omega(t)$

$$
\begin{gathered}
j(t)=\frac{\sqrt{\frac{I}{L}}\left(\omega_{m}-\omega_{c}\right)}{\operatorname{ch}\left[\frac{\alpha}{L}\left(\omega_{m}-\omega_{c}\right)\left(t-t_{0}\right)\right]} \\
\omega(t)=\omega_{c}-\left(\omega_{m}-\omega_{c}\right) \operatorname{th}\left[\frac{\alpha}{L}\left(\omega_{m}-\omega_{c}\right)\left(t-t_{0}\right)\right] .
\end{gathered}
$$

Pour $t=t_{0}$

$$
\begin{aligned}
& j=j_{\max }=\sqrt{\frac{I}{L}}\left(\omega_{m}-\omega_{c}\right) \\
& \omega=\omega_{c} .
\end{aligned}
$$

La constante d'intégration $t_{0}$ représente le temps séparant l'instant initial du moment pour lequel le courant est maximum.

L'évolution temporelle du courant et de la vitesse est représentée graphiquement sur la figure 5.

2.1.4 Principales grandeurs caractéristiques de la décharge. Nous nous proposons de mettre en évidence quelques grandeurs caractéristiques, fonction des conditions initiales. 


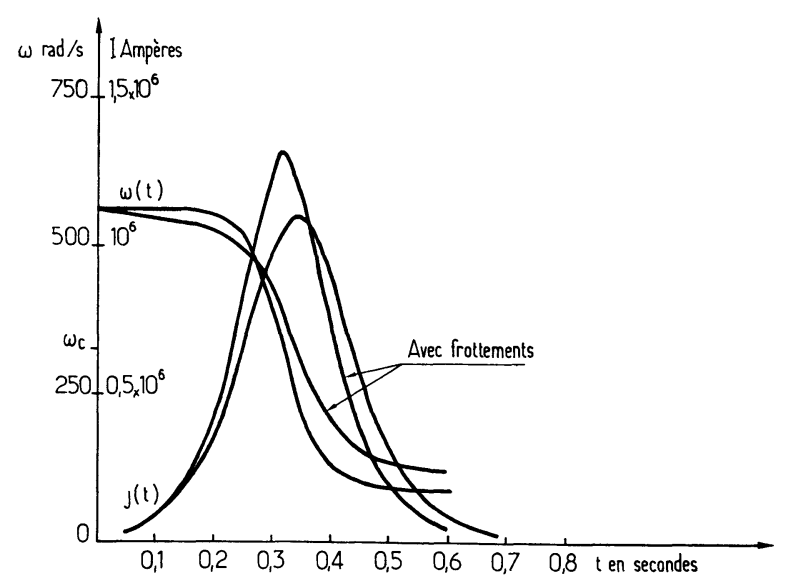

Fig. 5. - Evolution temporelle du courant $j(t)$ et de la vitesse $\omega(t)$. Influence des frottements.

- Quantité totale d'électricité $\int_{0}^{\infty} j(t) \mathrm{d} t$

$\int_{0}^{\infty} j(t) \mathrm{d} t \#$

$\# \int_{-\infty}^{+\infty} \sqrt{\frac{\bar{I}}{L}}\left(\omega_{m}-\omega_{c}\right) \frac{\mathrm{d} t}{\operatorname{ch}\left[\frac{\alpha}{L}\left(\omega_{m}-\omega_{c}\right)\left(t-t_{0}\right)\right]}=$ $=\frac{\pi}{\alpha} \sqrt{I \times L}$.

La quantité totale d'électricité est un invariant pour toutes les décharges.

- Temps moyen d'impulsion $\Delta t$

$$
\Delta t=\frac{\int_{0}^{\infty} j(t) \mathrm{d} t}{j_{m}}=\pi \frac{L}{\alpha}\left(\frac{1}{\omega_{m}-\omega_{c}}\right) .
$$

Ce temps est fonction de l'énergie totale mise en jeu. Cette propriété est liée à la non-linéarité des équations.

2.2 ÉVOLUTION DU SYSTÈME COMPORTANT DES FROTTEMENTS ET DES EFFETS D'ÉCRAN ÉLECTROMAGNÉTIQUE. - Nous nous proposons d'examiner les modifications du courant et de la vitesse dues aux frottements et aux effets d'écran que nous avions négligés précédemment.

2.2.1 Modification de l'évolution en présence d'effet d'écran. - La variation temporelle du champ magnétique $\mathrm{d} H / \mathrm{d} t$ crée dans les pièces métalliques de révolution des courants induits proportionnels à $-\mathrm{d} H / \mathrm{d} t$ qui s'opposent à la variation de ce champ. Ils diminuent le champ pendant la montée du courant dans génératrice, et tendent à le maintenir pendant sa décroissance. En pratique seul le rotor et les «bagues de court-circuit » des stators paraissent exercer un effet appréciable.

Appelons $\Phi$ et $\Phi_{e}$ le flux initial et le flux d'écran au travers du cercle des contacts, créés respectivement par le courant $j$ de la génératrice et par les courants induits. Si l'effet d'écran est suffisamment faible pour ne pas perturber la distribution initiale du champ, $\Phi$ est proportionnel à $j, \Phi_{e}$ est proportionnel à $\mathrm{d} j / \mathrm{d} t$ et nous pouvons écrire

$$
\frac{\Phi_{e}}{\Phi}=K \frac{1}{j} \frac{\mathrm{d} j}{\mathrm{~d} t}
$$

où $K$ est une constante faisant intervenir la géométrie du système.

Le flux utile est

$$
\Phi_{u}=\Phi-\Phi_{e}=\Phi\left(1-K \frac{1}{j} \frac{\mathrm{d} j}{\mathrm{~d} t}\right)
$$

La f. é. m. de la génératrice $u$ et le couple électromagnétique $C$ deviennent :

$$
\begin{aligned}
u=\frac{\Phi_{u}}{2 \pi} \omega & =\frac{\Phi}{2 \pi} \omega\left(1-K \frac{1}{j} \frac{\mathrm{d} j}{\mathrm{~d} t}\right) \\
= & \alpha \omega j-\beta \omega \frac{\mathrm{d} j}{\mathrm{~d} t} \\
C=-\frac{\Phi_{u}}{2 \pi} j & =-\frac{\Phi}{2 \pi} j\left(1-K \frac{1}{j} \frac{\mathrm{d} j}{\mathrm{~d} t}\right) \\
& =-\alpha j^{2}+\beta j \frac{\mathrm{d} j}{\mathrm{~d} t}
\end{aligned}
$$

où nous avons posé $\beta=K$. $\alpha$.

Les équations d'évolution s'écrivent maintenant :

$$
\begin{gathered}
L \frac{\mathrm{d} j}{\mathrm{~d} t}+R j-\alpha \omega j+\beta \omega \frac{\mathrm{d} j}{\mathrm{~d} t}=0 \\
I \frac{\mathrm{d} \omega}{\mathrm{d} t}+\alpha j^{2}-\beta j \frac{\mathrm{d} j}{\mathrm{~d} t}=0 .
\end{gathered}
$$

Les équations précédentes montrent que si les effets d'écran sont faibles, c'est-à-dire si $\beta \omega \ll L$ (nous verrons ultérieurement qu'il en est bien ainsi), les perturbations apportées à l'évolution sont du premier ordre. La valeur du courant maximum n'est pas changée, mais les deux versants de la courbe sont modifiés. Les effets d'écran allongent la montée et raccourcissent la descente de la courbe de courant. L'ordre de grandeur des effets d'écran a été déterminé expérimentalement. Nous donnons les résultats obtenus au chapitre suivant.

2.2.2 Modification de l'évolution en présence de frottements. - Afin de simplifier l'exposé nous négligeons les effets d'écran. Le circuit du mercure a été décrit précédemment. Le passage du mercure exerce sur le rotor un couple de frottements visqueux qui peut s'écrire, comme nous le verrons au paragraphe suivant $C=k \omega^{2}$, où $k$ est un coefficient lié à la géométrie du système et au coefficient de frottement $C_{f}$.

Le système d'équations d'évolution est maintenant :

$$
\begin{gathered}
L \frac{\mathrm{d} j}{\mathrm{~d} t}+R j-\alpha \omega j=0 \\
I \frac{\mathrm{d} \omega}{\mathrm{d} t}+\alpha j^{2}+k \omega^{2}=0 .
\end{gathered}
$$


Les courbes d'évolution temporelle du courant et de la vitesse sont modifiées par rapport aux courbes sans frottement. Les courbes de courant sont allongées vers la droite, les valeurs de l'intensité maximum sont plus faibles, tandis que les courbes de vitesse ne sont plus symétriques par rapport à la vitesse critique. Ces modifications sont représentées sur la figure 5 .

2.3 ÉVAluation de l'importance des PERTES PAR FROTTEMENTS. - Le circuit du mercure dans la génératrice a été décrit au premier chapitre, et il est représenté sur la figure 3 .

Le mercure injecté axialement à la pression $p_{0}$ ferme le circuit électrique entre le rotor et le stator, et permet à la génératrice de débiter un courant $j$. Dans la zone des contacts électriques, le courant est pratiquement normal aux faces en regard du stator et du rotor qui ont une résistivité beaucoup plus faible que le mercure, et il règne un champ magnétique $B$ de composantes $B_{r}, B_{\theta}, B_{z}$. Le champ azimutal $B_{\theta}$ est créé par le passage du courant dans le rotor, le stator crée les champs axial $B_{z}$ et radial $B_{r}$ (avec $B_{r} \ll B_{z}$ ). L'écoulement du mercure va alors se mettre en équilibre sous l'action des forces de pression, des forces de viscosité et des forces de Laplace $i \wedge B$. La densité de courant $i$, a priori non uniforme, est créée par le champ électrique dû à la génératrice, et par les champs électriques induits $V \wedge B, V$ étant la vitesse du fluide. Les efforts axiaux $i_{r} B_{\theta}-i_{\theta} B_{r}$ s'exercent dans le sens de l'écoulement, les efforts azimutaux $i_{r} B_{z}$ agissent dans le sens du couple électromagnétique c'est-à-dire en sens inverse du sens de rotation initial du rotor.

2.3.1 Expression du couple de frottement en l'absence de courant. - Lors du fonctionnement de la génératrice on ne peut dissocier le couple électromagnétique $\alpha j^{2}$ du couple de frottement visqueux agissant sur le rotor. La mesure de celui-ci, par la courbe de ralentissement du rotor, ne peut se faire qu'en l'absence de courant. Cette mesure permet de déterminer le coefficient de frottement $C_{f}$ et d'évaluer l'importance des frottements pendant le fonctionnement de la génératrice ; d'autant plus que le courant ne perturbe notoirement ce frottement que durant la seule pointe du courant.

L'étude de l'écoulement du mercure en l'absence de courant a été faite par J. Lucidarme [3] en considérant que :

- le système est de révolution,

- l'écoulement est établi (la longueur de l'écoulement $l$ étant grande par rapport à l'intervalle rotor stator $e$ ),

- le régime est stationnaire, c'est-à-dire que les variations de la vitesse du rotor sont faibles pendant le temps de transit du mercure dans les contacts.

Appelons :

$r:$ le rayon du rotor,

$e:$ l'intervalle moyen entre le rotor et le stator, $l$ : la longueur de l'écoulement suivant une génératrice du rotor,

$u$ : la vitesse périphérique du rotor,

$V:$ la vitesse du fluide,

$\rho$ : la masse spécifique du mercure,

$v$ : la viscosité cinématique du mercure.

Le nombre de Reynolds de l'écoulement est

$$
R=\frac{V . e}{v} .
$$

L'ordre de grandeur des vitesses d'écoulement est $V \simeq 20 \mathrm{~m} / \mathrm{s}, \quad e=1,5 \times 10^{-3} \mathrm{~m}, \quad v=10^{-7}$ d'où $R \simeq 3 \times 10^{5}$.

L'écoulement est turbulent rugueux, les couches laminaires sont fines par rapport à l'épaisseur totale de la veine; par suite, le profil des vitesses est assez carré. Appelons $C_{f}$ le coefficient de frottement pour un régime turbulent rugueux.

Il résulte de cette étude que le fluide, par suite des frottements visqueux, prend une vitesse azimutale $V_{\theta}(z)$ fonction du rapport de la vitesse périphérique $\mathrm{du}$ rotor à la vitesse moyenne axiale du fluide $V_{z}$ qui est constante :

$$
V_{\theta}(z)+\frac{u}{2}\left[1-\exp \left(-C_{f} \frac{u}{V_{z}} \cdot \frac{z}{e}\right)\right] .
$$

La force de frottement azimutale par unité de surface sur le rotor est alors :

$$
\tau=\frac{1}{2} \rho C_{f}\left(u-V_{\theta}\right)^{2}
$$

d'où l'expression du couple de frottement :

$$
C=\pi r^{2} \rho C_{f} \int_{0}^{l}\left(u-V_{\theta}\right)^{2} \mathrm{~d} z .
$$

On obtient, tout calcul fait en posant

$$
\psi=C_{f} \frac{u}{V_{z}} \cdot \frac{l}{e} .
$$

$$
\begin{aligned}
C=\frac{1}{4} & \pi r^{2} l \rho C_{f} u^{2} \times \\
& \times\left[1+\frac{1}{\psi}\left(5+\exp \frac{-\psi}{2}\right)\left(1-\exp \frac{-\psi}{2}\right)\right] .
\end{aligned}
$$

Dans le cas où $V_{z} \ll u$, c'est-à-dire dans le cas des faibles pressions d'injection ou des grandes vitesses de rotation $\mathrm{du}$ rotor

$$
C=C_{0}=\frac{1}{4} \pi r^{2} l \rho C_{f} u^{2}
$$

ce qui traduit un frottement en $(u / 2)^{2}$ qui signifie que tout le fluide est mis instantanément en rotation à la vitesse $u / 2$.

- Dans le cas où $V_{z} \gg u$, c'est-à-dire dans le cas des fortes pressions d'injection ou des faibles vitesses de rotation du rotor, on a : $C=4 C_{0}$ (ce qui signifie 
que le fluide ne prend qu'une vitesse azimutale faible devant celle de la paroi).

2.3.2 Limite supérieure du couple de frottement en présence de courant. - En présence de courant et de champ magnétique l'ordre de grandeur du nombre de Reynolds est comme précédemment $R \simeq 3 \times 10^{5}$.

Le nombre de Hartmann de l'écoulement est

$$
M=B \cdot e \cdot(\eta \cdot \rho \cdot v)^{-1 / 2}
$$

où $B \simeq 3 \mathrm{~T}$ ordre de grandeur des champs magnétiques,

$\eta=10^{-6} \Omega . \mathrm{m}$ résistivité du mercure

d'où $M \simeq 10^{2}$ et $R / M \simeq 3 \times 10^{3}$. Or Lazarus [4] a trouvé que pour $R / M \geqslant 900$, l'écoulement restait turbulent et rugueux et n'était pas influencé notablement par la présence du champ magnétique, ce qui est notre cas.

$\mathrm{Au}$ début du fonctionnement de la génératrice, la vitesse de rotation est élevée et le courant $j$ est faible. Les forces de viscosité provoquent la mise en rotation du mercure comme en l'absence de courant. Ensuite l'impulsion de courant se développe tandis que la vitesse chute, les forces de Laplace $i \wedge B$ deviennent importantes, celles-ci s'exercent en sens contraire du sens de rotation du rotor. Un calcul simple [5] montre qu'en l'absence de forces de viscosité le mercure prendrait une vitesse azimutale finale de l'ordre de $-u / 2$ pour un courant $j=1 \mathrm{MA}$, dans ce cas la vitesse du rotor est de l'ordre de la vitesse critique. Si toute la veine de mercure prenait instantanément cette vitesse $V_{\theta}=-u / 2$, le couple de frottement correspondant serait $C=9 C_{0}$, cette valeur représente la limite supérieure du couple de frottement, qui n'est pas atteinte en pratique.

$\mathrm{Au}$ chapitre suivant nous allons déterminer expérimentalement en l'absence de courant le couple de frottement du mercure sur le rotor, et nous en déduirons la valeur $\mathrm{du}$ coefficient de frottement $C_{f}$. Nous verrons qu'en présence de courant ce couple devient négligeable devant le couple électromagnétique dès que le courant $j$ est supérieur à $200000 \mathrm{~A}$.

Cependant dans le cas où $j$ est faible, c'est-à-dire en pratique pendant le temps d'amorçage de la génératrice, la vitesse de rotation est élevée et le freinage du rotor dû aux forces de frottement visqueux entraîne une perte sensible de l'énergie cinétique utile

$$
\frac{I}{2}\left(\omega_{m}-\omega_{c}\right)^{2}
$$

emmagasinée dans le volant.

2.4 VALEURS DES PARAMÈTRES DE CONSTURUCTION. Nous donnons ci-dessous les valeurs calculées [6] de la génératrice réalisée.

Masse du rotor ......... $173 \mathrm{~kg}$

Energie cinétique maximum ... $5 \mathrm{MJ}$
Vitesse périphérique maximum

$\mathrm{du}$ rotor (aux contacts liquides) $120 \mathrm{~m} / \mathrm{s}$

Couple de torsion admissible... $10^{5} \mathrm{~m} . \mathrm{N}$

Intensité du courant correspon-

dant au couple maximum....

Résistance interne de la généra-

trice ..................

Coefficient de couplage électro-

mécanique............... $\alpha=19,1 \times 10^{-9} \mathrm{H}$

Moment d'inertie du rotor.... I I $=5,14$

3. Étude expérimentale de la dynamo unipolaire. L'expérimentation systématique de la dynamo unipolaire impulsionnelle a été effectuée en court-circuit et avec différentes charges: une charge purement résistive d'environ $3 \times 10^{-6} \Omega$ constituée d'une plaque d'acier placée aux bornes de la génératrice, une charge selfique $\left(L \simeq 10^{-6} \mathrm{H}, R \simeq 4,5 \times 10^{-6} \Omega\right)$ constituée par une bobine spéciale à champ fort [6].

Nous allons tout d'abord décrire les moyens de mesure, puis nous nous proposons de montrer quelques courbes typiques obtenues et de déterminer le paramètre de fonctionnement de la génératrice. Les nombreux essais effectués ont mis en évidence le rôle important de la pression d'injection du mercure dans la commutation des hautes intensités. Nous allons enfin décrire les phénomènes observés lorsque cette pression d'injection est trop faible et donner un critère expérimental de stabilité des contacts liquides.

3.1 Les MOYENS DE MESURE. - Un ensemble de moyens, que nous décrivons succinctement ci-dessous, permet la mesure des performances de la génératrice.

3.1.1 Mesure des grandeurs mécaniques. a) Mesure de la vitesse. - Une dynamo tachymétrique montée en bout du rotor mesure la vitesse instantanée de rotation. Ce tachymètre est une petite dynamo unipolaire à aimants permanents possédant une caractéristique linéaire et un temps de réponse très court. Le signal obtenu est envoyé sur un oscilloscope et photographié.

b) Mesure du couple. - Le moment d'inertie du rotor étant connu avec précision, le couple électromagnétique se calcule à partir de la courbe de ralentissement du rotor :

$$
C=I \frac{\mathrm{d} \omega}{\mathrm{d} t}
$$

3.1.2 Mesure des grandeurs électriques. a) Mesure du courant global. - La mesure du courant global est réalisée à l'aide d'une sonde de Rogowski. Cette sonde est constituée par un solénoïde long, de faible diamètre comportant un grand nombre de spires de fil fin. Elle est installée dans une encoche entre les bornes de la machine de façon à ce que l'axe du solénoïde soit perpendiculaire au courant. La force électromotrice induite étant proportionnelle à $\mathrm{d} B / \mathrm{d} t$, c'està-dire à $\mathrm{d} j / \mathrm{d} t$, la mesure de la tension $u(t)$ prise aux 
bornes de la sonde donne la valeur instantanée de $\mathrm{d} j / \mathrm{d} t$.

La mesure du courant global $j(t)$ s'obtient par intégration graphique de la courbe $\mathrm{d} j / \mathrm{d} t$ enregistrée photographiquement sur un oscilloscope.

b) Mesure de la force électromotrice. - Deux sondes électriques en contact avec les bagues de courtcircuit des stators donnent la tension existante entre ces deux bagues. Elles mesurent la f. é. $m$. de la génératrice, la chute résistive de tension dans le rotor étant très faible.

3.1.3 Mesures sur l'injection du mercure - Les paramètres du fonctionnement liés au circuit du mercure sont :

la pression d'injection du mercure,

le volume de mercure injecté.

a) Mesure du volume injecté. - Le volume de mercure injecté est déterminé par le réglage de la position initiale du piston dans les vérins.

b) Mesure de la pression. - Un manomètre placé sur une bouteille d'air comprimé mesure la pression initiale d'injection. La pression d'injection chute légèrement au cours de la détente, car la bouteille d'air comprimé a un volume fini. Pour déterminer cette chute de pression, un capteur de pression a été placé à la sortie d'un vérin.

c) Mesure du remplissage des contacts. - Ce montage n'a été utilisé que lors du début de l'expérimentation de la génératrice, il consiste en deux sondes électriques plongeant dans les intervalles rotor-stator près des étranglements. Elles indiquent la qualité du remplissage en mercure des contacts tournants.

3.2 IMPORTANCE DES FROTTEMENTS. - 3.2.1 Mesure $d u$ couple de frottements en l'absence de courant. La génératrice ne débitant aucun courant, nous avons enregistré le ralentissement du rotor dû aux forces de frottements visqueux lors de l'injection du mercure dans les contacts, dans les conditions initiales suivantes :

Vitesse initiale du rotor ......... $7000 \mathrm{tr} / \mathrm{mn}$ Pression d'injection de mercure ..... $80 \mathrm{kgf} / \mathrm{cm}^{2}$ Volume de mercure injecté ....... 91 par vérin Durée de l'injection ............ 1,5 s

Nous en déduisons la vitesse axiale du fluide $V_{z}=8,5 \mathrm{~m} / \mathrm{s}$ et la vitesse périphérique $\mathrm{du}$ rotor $u=55 \mathrm{~m} / \mathrm{s}$.

La courbe $\omega(t)$ enregistrée est pratiquement une droite dont la pente est $\mathrm{d} \omega / \mathrm{d} t=-70 \mathrm{rad} / \mathrm{s}$. Le couple de frottement correspondant

$$
I \frac{\mathrm{d} \omega}{\mathrm{d} t}=360 m . \mathrm{N}
$$

3.2.2 Evaluation $d u$ coefficient $C_{f}$. - La relation (11) permet de déterminer le coefficient de frotte- ment pour un régime turbulent rugueux $C_{f}$. Nous considérons que la partie du mercure injecté qui s'introduit dans l'intervalle rotor-stator compris entre les injecteurs prend la vitesse azimutale $u / 2$, et qu'elle exerce sur le rotor un couple de frottement qui s'exprime par la relation (12).

Nous obtenons, tout calcul effectué : $C_{f}=4 \times 10^{-3}$. Ce chiffre correspond bien aux valeurs expérimentales usuelles.

3.2.3 Evaluation de la limite supérieure du couple de frottement en présence de |courant. - Nous avons vu que la limite supérieure du couple de frottement est

$$
C=9 C_{0}=\frac{9}{4} \pi r^{2} l \rho C_{f} u^{2}
$$

lorsque le courant est de l'ordre de $1 \mathrm{MA}$ et la, vitesse de rotation voisine de la vitesse critique. Dans les cas pratiques $\omega_{c} \leqslant 500 \mathrm{rad} / \mathrm{s}$, en prenant pour $C_{f}$ la valeur $4 \times 10^{-3}$ déterminée précédemment, nous obtenons $C \simeq 500 \mathrm{~m} . \mathrm{N}$. Or, pour $1 \mathrm{MA}$ le couple électromagnétique vaut $2 \times 10^{4} \mathrm{~m}$. N. Le couple de frottement est négligeable devant le couple électromagnétique.

3.3 EXPÉRIMENTATION DE LA GÉNÉRATRICE EN COURTCIRCUIT. - L'intérêt de l'expérimentation de la génératrice en court-circuit est qu'elle permet de déterminer les caractéristiques internes qui lors d'essais en charge ne peuvent être dissociées de celle de la charge.

3.3.1 Courbes typiques de l'évolution en courtcircuit. - La figure 6 représente les évolutions

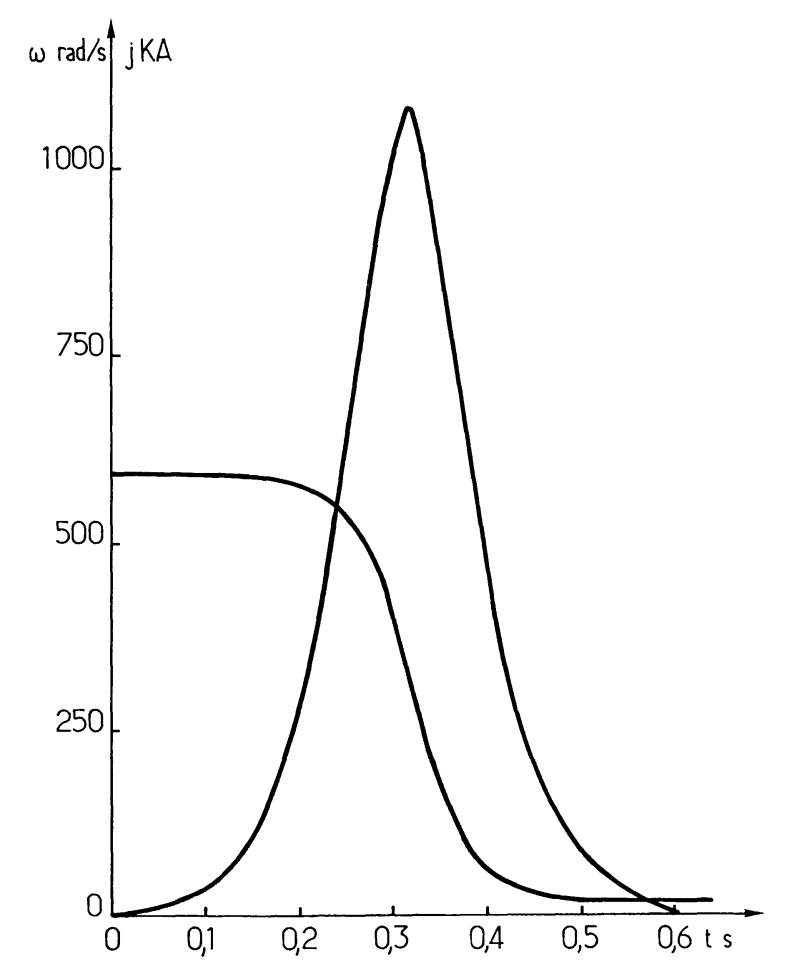

FIG. 6. - Evolution temporelle de la vitesse et du courant. Essai 162 en court-circuit. 
temporelles de la vitesse du rotor $\omega(t)$ et du courant $j(t)$, pour un essai en court-circuit à partir des conditions initiales suivantes :

Vitesse du rotor $5660 \mathrm{tr} / \mathrm{mn}$ Pression d'injection du mercure .... $100 \mathrm{kgf} / \mathrm{cm}^{2}$ Volume de mercure injecté ....... 51 par vérin.

La courbe de chute de vitesse est régulière et symétrique par rapport à la vitesse critique $\omega_{c}=310 \mathrm{rad} / \mathrm{s}$.

Le couple électromagnétique maximum est

$$
I\left(\frac{\mathrm{d} \omega}{\mathrm{d} t}\right)_{m}=2,3 \times 10^{4} \mathrm{~m} \cdot \mathrm{N} .
$$

La courbe de l'impulsion de courant $j(t)$ est obtenue par intégration graphique du signal proportionnel à $\mathrm{d} j / \mathrm{d} t$ délivré par la sonde de Rogowsky. Le courant maximum est $j_{m}=1,08 \times 10^{6} \mathrm{~A}$.

Par élimination graphique du temps entre les courbes précédentes, nous avons tracé figure 7 ; le diagramme d'évolution dans le plan $(\omega, j)$. La courbe obtenue est pratiquement une ellipse, comme la théorie le prévoit.

3.3.2 Evaluation des effets d'écran électromagnétique. - L'équation d'évolution mécanique (7) permet d'évaluer le coefficient d'effet d'écran $\beta$.

En effet, pour deux points $A$ et $B$ de l'évolution correspondant à un même courant $j_{A}=j_{B}$, nous pouvons écrire en chacun de ces points :

$$
\begin{aligned}
& I\left(\frac{\mathrm{d} \omega}{\mathrm{d} t}\right)_{A}+\alpha j_{A}^{2}-\beta j_{A}\left(\frac{\mathrm{d} j}{\mathrm{~d} t}\right)_{A}=0 \\
& I\left(\frac{\mathrm{d} \omega}{\mathrm{d} t}\right)_{B}+\alpha j_{B}^{2}-\beta j_{B}\left(\frac{\mathrm{d} j}{\mathrm{~d} t}\right)_{B}=0
\end{aligned}
$$

par différence nous éliminons les termes $\alpha j_{A}^{2}$ et $\alpha j_{B}^{2}$ d'où

$$
\beta=\frac{I\left[\left(\frac{\mathrm{d} \omega}{\mathrm{d} t}\right)_{A}-\left(\frac{\mathrm{d} \omega}{\mathrm{d} t}\right)_{B}\right]}{j_{A}\left[\left(\frac{\mathrm{d} j}{\mathrm{~d} t}\right)_{A}-\left(\frac{\mathrm{d} j}{\mathrm{~d} t}\right)_{B}\right]} .
$$

Le moment d'inertie $I$ du rotor étant connu avec précision, nous avons déterminé $\beta$ à partir des courbes expérimentales de la figure 6 . Nous avons mesuré aux points correspondant à $j=5 \times 10^{5} \mathrm{~A}$ et $j=7 \times 10^{5} \mathrm{~A}, \beta=0,19 \mathrm{H} \times \mathrm{s}$ et $\beta=0,18 \mathrm{H} \times \mathrm{s}$.

Aux erreurs de mesure près, nous trouvons la même valeur $\beta \simeq 0,2 \times 10^{-9} \mathrm{H} \times \mathrm{s}$. Les mêmes mesures effectuées sur d'autres courbes expérimentales donnent un résultat analogue. Les éq. (6) et (7) sont donc une bonne approximation de l'évolution de la génératrice.

Par définition du coefficient $\beta$, le rapport du flux d'écran au travers du cercle des contacts au flux créé par le stator est

$$
\frac{\Phi_{e}}{\Phi}=\frac{\beta}{\alpha} \cdot \frac{1}{j} \frac{\mathrm{d} j}{\mathrm{~d} t} .
$$

Or $\alpha \simeq 20 \times 10^{-9} \mathrm{H}$, d'où

$$
\frac{\Phi_{e}}{\Phi}=10^{-2} \frac{1}{j} \frac{\mathrm{d} j}{\mathrm{~d} t} \text {. }
$$

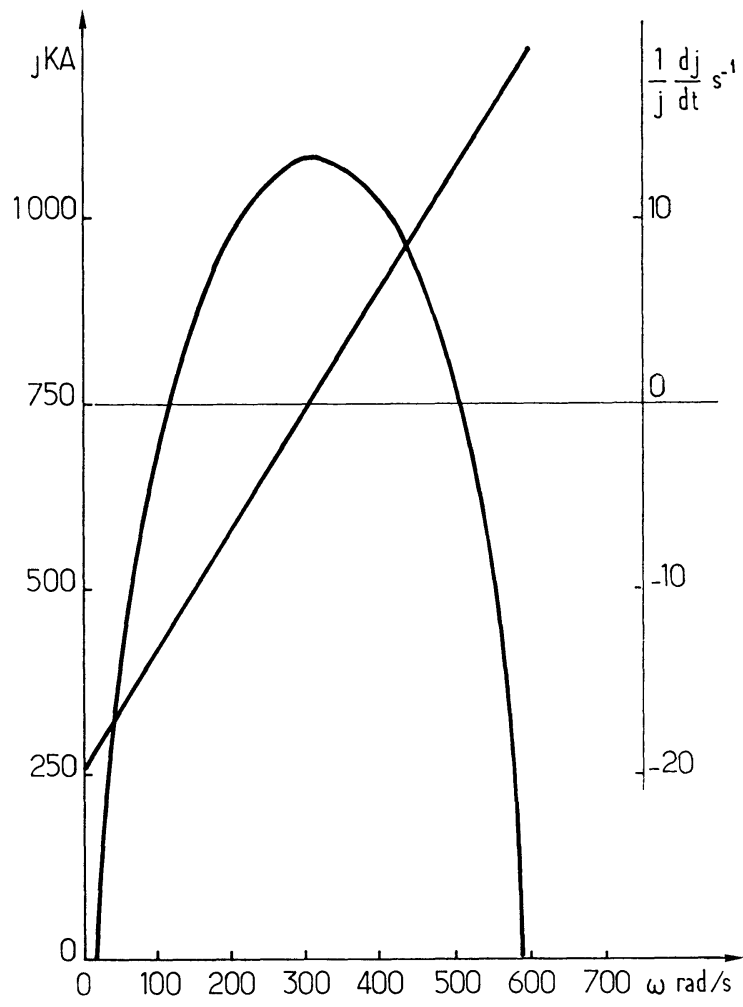

Fig. 7. - Diagramme d'évolution en $\omega, j$. Essai 162 en courtcircuit.

La variation de $\Phi_{e} / \Phi$ au cours de l'évolution est représentée par la variation de $(1 / j)(\mathrm{d} j / \mathrm{d} t)$ graduée en $\%$. Sa valeur maximum en court-circuit est

$$
\left(\frac{\Phi_{e}}{\Phi}\right)_{\max } \simeq 20 \%
$$

est atteinte au début et à la fin de l'impulsion de courant.

Lors du fonctionnement de la génératrice débitant sur une charge selfique adaptée, les temps sont allongés et les effets d'écran sont réduits d'un facteur 2 environ. Par la suite nous négligerons donc les effets d'écran électromagnétique.

3.3.3 Mesure des paramètres de fonctionnement. Les effets d'écran et les frottements étant négligeables après l'amorçage de la génératrice, les courbes expérimentales $\omega(t)$ et $j(t)$ permettent de déterminer les paramètres de fonctionnement de la génératrice : la résistance interne $R$, la self interne $L$ et le coefficient de couplage électromécanique $\alpha$.

En effet la variation de

$$
\frac{1}{j} \frac{\mathrm{d} j}{\mathrm{~d} t}=\frac{\alpha}{L} \omega-\frac{R}{L}
$$

en fonction de $\omega$ est une droite dont l'ordonnée à l'origine est $-R / L$, l'intersection avec l'axe des $\omega$ a lieu pour $\omega_{c}=R / \alpha$. La construction point par point de cette droite à partir des courbes expérimentales donne les valeurs $-R / L$ et $R / \alpha$. 
Le coefficient $\alpha=-\frac{I}{j^{2}} \frac{\mathrm{d} \omega}{\mathrm{d} t}$ est déduit de la mesure du couple maximum $C_{m}=-I(\mathrm{~d} \omega / \mathrm{d} t)_{m}$ et du courant maximum $j_{m}$.

Pour de nombreux essais, nous avons déterminé ainsi les paramètres de fonctionnement.

Considérons l'essai en court-circuit pour lequel nous avons déterminé précédemment le coefficient d'effet d'écran $\beta$.

Nous avons tracé figure 7 la variation de $(1 / j)(\mathrm{d} j / \mathrm{d} t)$ en fonction de $\omega$.

Nous obtenons :

$$
\begin{aligned}
& \omega_{c}=\frac{R}{\alpha}=305 \mathrm{rad} / \mathrm{s} \\
& \frac{R}{L}=20 \mathrm{~s}^{-1} \\
& j_{m}=1,08 \times 10^{6} \mathrm{~A} \\
& C_{m}=2,3 \times 10^{4} \mathrm{~m} \cdot \mathrm{N}
\end{aligned}
$$

d'où nous déduisons :

$$
\begin{gathered}
\alpha=19,7 \times 10^{-9} \mathrm{H} ; \\
R=6 \times 10^{-6} \Omega ; \quad L=0,3 \times 10^{-6} \mathrm{H} .
\end{gathered}
$$

Ces valeurs sont voisines des valeurs théoriques données à la fin du chapitre 1. Seule la résistance interne de la machine semble élevée. D'autres mesures effectuées à partir d'essais en court-circuit donnent des valeurs voisines de celles-ci; la dispersion des résultats indique que la précision de la mesure de ces paramètres est d'environ $20 \%$.

3.4 EXPÉRIMENTATION DE LA GÉNÉRATRICE EN CHARGE. - 3.4.1 Courbes typiques de l'évolution en charge. - Les enregistrements photographiques de la figure 8 correspondent respectivement à un essai sur charge résistive de $3 \times 10^{-6} \Omega$ et à un essai sur charge selfique. La charge selfique est une bobine spéciale à champ fort [6], La self de cette bobine n'est connue
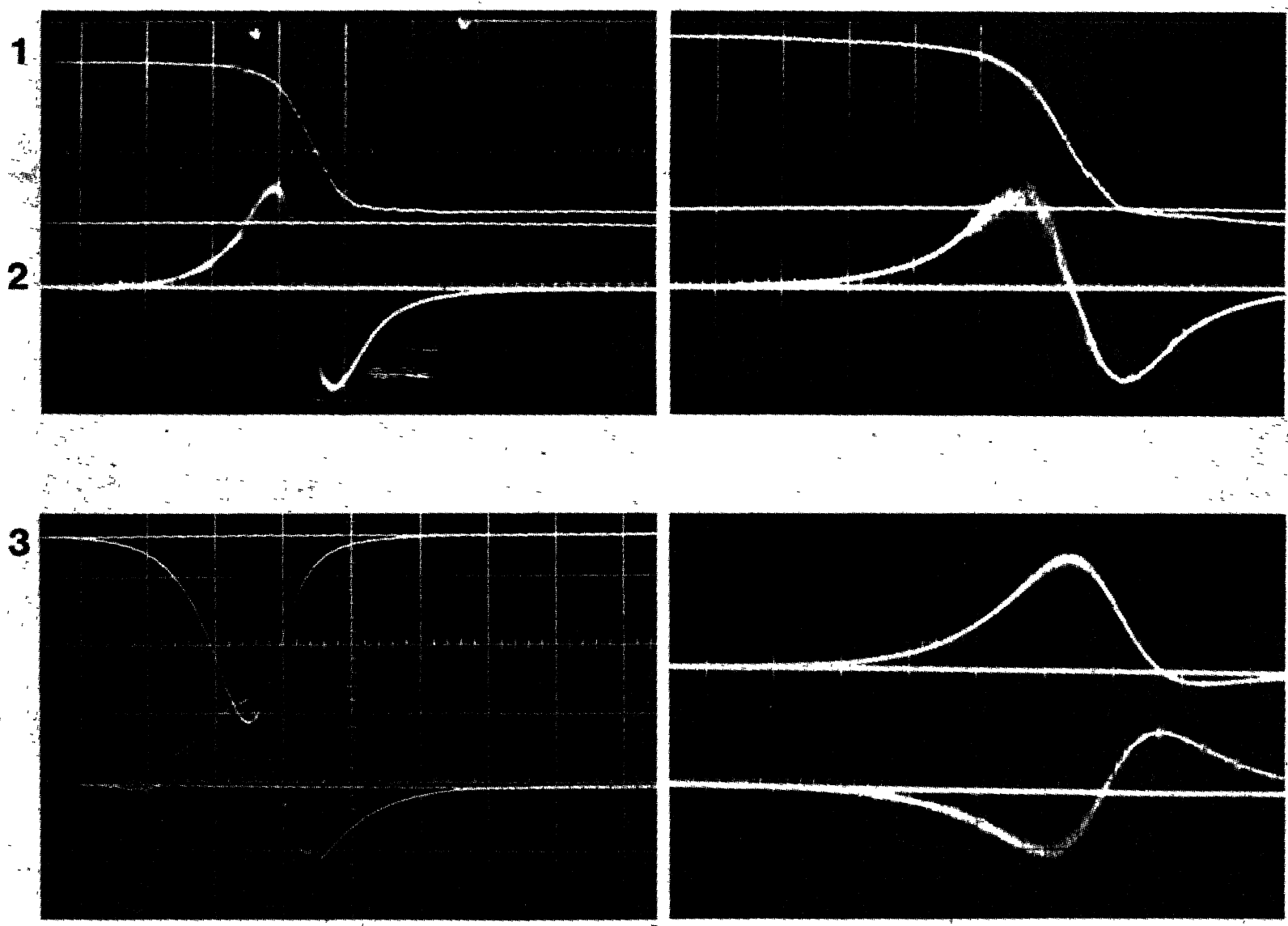

FIG. 8.

balayage $0,1 \mathrm{~s} / \mathrm{cm}$

Essai $n^{\circ} 100$ sur charge de $3 \mu \Omega$

vitesse initiale ............... $8000 \mathrm{tr} / \mathrm{mn}$

pression d'injection $\ldots \ldots \ldots \ldots . \quad 140 \mathrm{~kg} / \mathrm{cm}^{2}$

volume injecté $\ldots \ldots \ldots \ldots \ldots \ldots . .2 \times 51$

courant obtenu ............. 1,4 MA balayage $0,1 \mathrm{~s} / \mathrm{cm}$ Essai $n^{\circ} 199$ sur charge selfique

vitesse initiale .............. $11500 \mathrm{tr} / \mathrm{mn}$ pression d'injection ........... $185 \mathrm{~kg} / \mathrm{cm}^{2}$ volume injecté $\ldots \ldots \ldots \ldots \ldots \ldots .2 \times 91$ courant obtenu $\ldots \ldots \ldots \ldots \ldots \ldots . \quad 1,335 \mathrm{MA}$

Evolution temporelle de la vitesse ......... 1

de la dérive du courant . 2

de la f. é. m......... 3 
qu'approximativement étant donné sa structure complexe: $L \sim 10^{-6} \mathrm{H}, R \sim 4,5 \times 10^{-6} \Omega$.

De haut en bas, les courbes représentent l'évolution de la vitesse, de la dérivée du courant et de la force électromotrice. Les conditions initiales de fonctionnement et les courants maximums obtenus sont indiqués sur la figure. L'allure générale des courbes n'est pas modifiée par rapport aux courbes obtenues en court-circuit. On observe pour l'essai sur charge selfique que, conformément à la théorie, la vitesse de rotation s'inverse durant l'impulsion lorsque la vitesse initiale est supérieure au double de la vitesse critique. D'autre part, nous vérifions bien que la largeur de l'impulsion n'est fonction que de la self totale du circuit et du courant maximum.

3.4.2 Mesure des paramètres de fonctionnement. - Nous avons déterminé, en utilisant la même méthode que précédemment pour les essais en courtcircuit, les valeurs des paramètres de fonctionnement :

a) Fonctionnément sur chargé résistive de $3 \mu \Omega$. Pour l'essai 100 reproduit sur la figure; nous mesurons :

$$
\begin{aligned}
& \omega_{c}=440 \mathrm{rad} / \mathrm{s} \\
& j_{\max }=1,4 \times 10^{6} \mathrm{~A} \\
& C_{\max }=3,6 \times 10^{4} \mathrm{~m} \cdot \mathrm{N} \\
& \frac{R}{L}=28 \mathrm{~s}^{-1}
\end{aligned}
$$

d'où :

$$
\begin{gathered}
\alpha=18,5 \times 10^{-9} \mathrm{H}, \\
R=8,1 \times 10^{-6} \Omega, \quad L=0,29 \times 10^{-6} \mathrm{H} .
\end{gathered}
$$

b) Fonctionnement sur charge selfique - Pour l'essai 199 de la figure, nous mesurons :

$$
\begin{aligned}
& \omega_{c}=460 \mathrm{rad} / \mathrm{s} \\
& j_{\max }=1,35 \times 10^{6} \mathrm{~A} \\
& C_{\max }=3,8 \times 10^{4} \mathrm{~m} \cdot \mathrm{N} \\
& \frac{R}{L}=8 \mathrm{~s}^{-1}
\end{aligned}
$$

d'où :

$$
\begin{gathered}
\alpha=21 \times 10^{-9} \mathrm{H}, \\
R=9,7 \times 10^{-6} \Omega, \quad L=1,2 \times 10^{-6} \mathrm{H} .
\end{gathered}
$$

Ces valeurs correspondent aux valeurs théoriques, aux erreurs de mesures près.

3.5 Performances maximum obtenues. - Nous indiquons ci-dessous les performances maximums obtenues actuellement pour les trois charges utilisées.
3. 6 INFLUENCE DE LA PRESSION D'INJECTION DU MERCURE, INSTABILITÉS DES QQONTAĊTS LIQUIDES. - Lors de l'expérimentation systématique de la génératrice il est apparu que la pression d'injection du mercure nécessaire au bon fonctionnement des balais liquides croissait avec la vitesse initiale. Lorsque la pression d'injection est insuffisante, le fonctionnement de la génératrice s'accompagne d'un bruit important. Des instabilités graves apparaissent, elles engendrent des efforts électromagnétiques et des vibrations mécaniques intenses, allant jusqu'à provoquer la détérioration rapide des paliers supportant le rotor dans le cas où cette pression d'injection est trop insuffisante.

3.6.1 Etude expérimentale du développement des instabilités - Trois essais en court-circuit montrent clairement l'apparition des instabilités lorsque l'on baisse la pression d'injection (Fig. 9). Ces trois essais ont été effectués dans les mêmes conditions initialès de vitesse $(5100 \mathrm{tr} / \mathrm{mn})$ et de volume de mercure injecté (5 1 par vérin) mais avec des pressions d'injection décroissantes.

Essai $158 \ldots \ldots \ldots \ldots \ldots \ldots \ldots 65 \mathrm{kgf} / \mathrm{cm}^{2}$

Essai $159 \ldots \ldots \ldots \ldots \ldots \ldots \ldots 60 . \ldots \ldots f / \mathrm{cm}^{2}$

Essai $156 \ldots \ldots \ldots \ldots \ldots \ldots \ldots 5 \mathrm{kgf} / \mathrm{cm}^{2}$.

Pour ces trois essais, le courant maximum obtenu est le même : $920000 \mathrm{~A}$ ainsi que le couple maximum $1,65 \times 10^{4} \mathrm{~m} . \mathrm{N}$.

Des instabilités se développent dès que la pression d'injection est inférieure à $65 \mathrm{kgf} / \mathrm{cm}^{2}$. Elles se manifestent simultanément sur les différentes courbes d'évolution que nous allons analyser en détail. Par la suite nous appelons pression critique, la pression limite en dessous de laquelle apparaissent les instabilités.

a) Evolution du courant. - Les courbes de la dérivée du courant par rapport au temps indiquent un fonctionnement correct de la génératrice jusqu'au voisinage du maximum de courant. Les valeurs du courant maximum obtenu correspondent au calcul théorique et sont indépendantes de la pression d'injection. Après le maximum du courant, et parfois juste à ce maximum, il apparaît de brusques variations de la dérivée du courant. Les amplitudes de ces variations sont d'autant plus grandes que la pression est inférieure à la pression critique, elles sont toujours dirigées du côté négatif de la dérivée et correspondent par conséquent à une chute du courant. On remarque d'autre part que l'impulsion de courant s'élargit légèrement et ceci d'autant plus que la pression est insuffisante.

b) Evolution de la vitesse. - Les courbes de l'évolution temporelle de la vitesse sont identiques pour les

\begin{tabular}{lcccc}
\multicolumn{1}{c}{ Charge } & $\begin{array}{c}\text { Vitesse } \\
\text { initiale }\end{array}$ & $\begin{array}{c}\text { Vitesse } \\
\text { finale }\end{array}$ & $\begin{array}{c}\text { Courant } \\
\text { maximum }\end{array}$ & $\begin{array}{c}\text { Energie } \\
\text { dissipée }\end{array}$ \\
Court-circuit & $\overline{-}$ & $\overline{0}$ & $1,28 \times 10^{6} \mathrm{~A}$ & $1,0 \overline{\mathrm{MJ}}$ \\
$3 \mu \Omega$ & $6200 \mathrm{tr} / \mathrm{mn}$ & $570 \mathrm{tr} / \mathrm{mn}$ & $1,52 \times 10^{6} \mathrm{~A}$ & $1,87 \mathrm{MJ}$ \\
Bobine à champ fort & $8160 \mathrm{tr} / \mathrm{mn}$ & $-1200 \mathrm{tr} / \mathrm{mn}$ & $1,35 \times 10^{6} \mathrm{~A}$ & $3,7 \mathrm{MJ}$
\end{tabular}



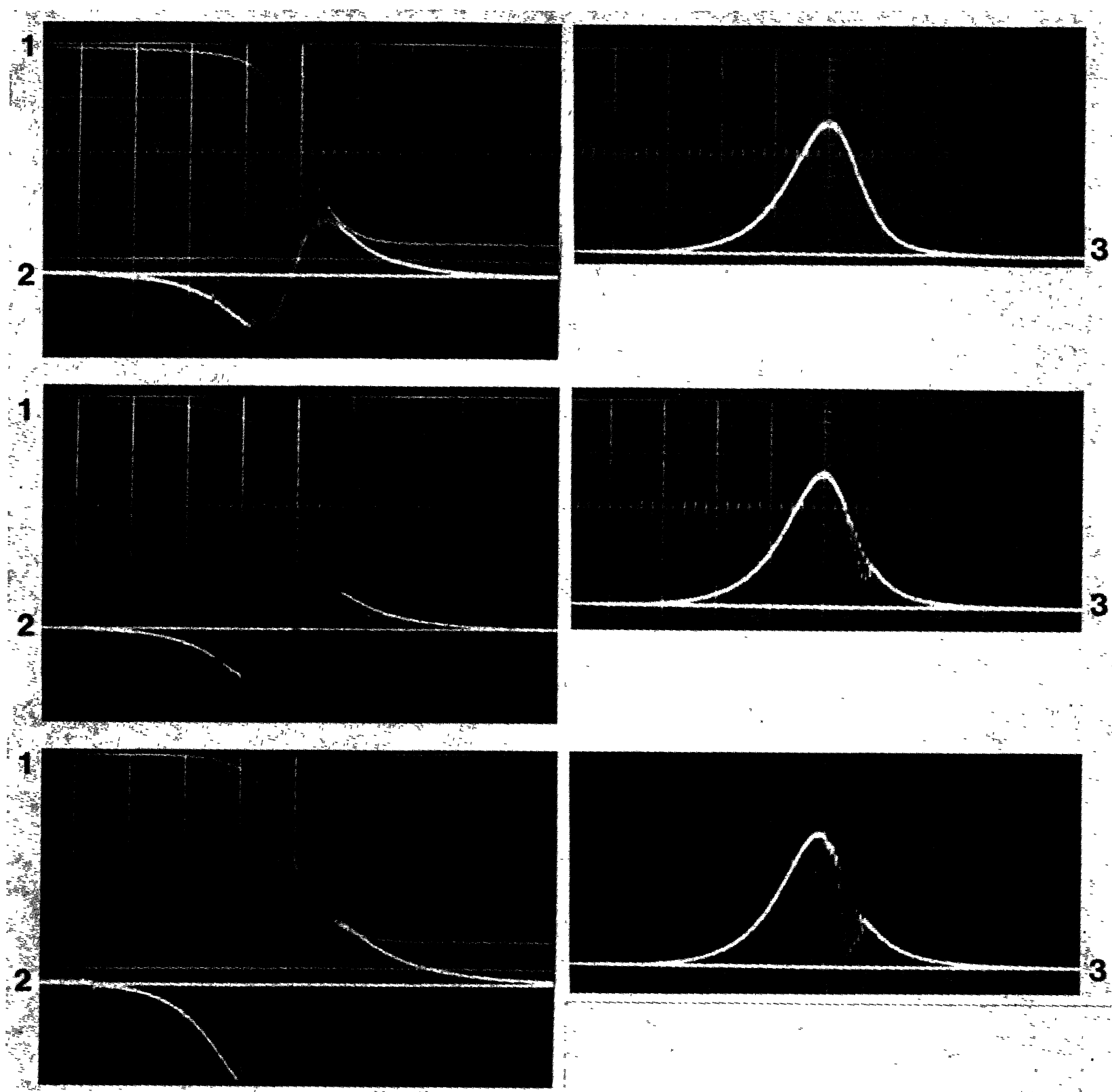

Fig. 9. - Influence de la pression d'injection du mercure sur l'évolution de la vitesse (1), de la dérivée du courant (2) et de la f. é. m. (3). Essais en court-circuit.

vitesse initiale ....................5100 $\mathrm{tr} / \mathrm{mn}^{-1}$ courant maximum ...............920 kA

trois essais de la figure 9 jusqu'à la vitesse critique, les couples maximum mesurés sont les mêmes. Lors de l'apparition des instabilités, on observe une légère rupture de pente, nettement visible pour l'essai 156 , elle correspond à une diminution du couple électromagnétique. Pour une même vitesse initiale, la vitesse finale est d'autant plus élevée que l'amplitude de l'instabilité a été forte :

$400 \mathrm{tr} / \mathrm{mn}$ pour l'essai 158

$530 \mathrm{tr} / \mathrm{mn}$ pour l'essai 159

$630 \mathrm{tr} / \mathrm{mn}$ pour l'essai 156 .

c) Evolution de la tension. - Les deux sondes électriques en contact avec les bagues de court-circuit pression décroissante de haut en bas. . . 65 bars, 60 bars, 55 bars balayage $\ldots \ldots \ldots \ldots \ldots \ldots \ldots \ldots, 1 \mathrm{~s} / \mathrm{cm}^{1}$

du stator mesurent la force électromotrice de la machine. L'évolution de ces courbes sont identiques jusqu'à l'apparition des instabilités, on observe alors un développement progressif d'oscillations, situées en dessous de la courbe de tension, qui s'amortissent ensuite rapidement.

Pour des essais effectués à partir de conditions initiales différentes, la fréquence de ces oscillations apparaît indépendante de la pression d'injection, de la vitesse $\mathrm{du}$ rotor et du courant, elle reste toujours constante et de l'ordre de $155 \mathrm{~Hz}$. L'amplitude de ces oscillations est par contre croissante avec l'écart absolu séparant la pression d'injection de la pression critique. 
d) Indications données par les sondes de remplissage. - Le signal délivré par les sondes plongeant dans le mercure des balais liquides est, lors du bon fonctionnement de la génératrice, identique à la tension recueillie entre les bagues de court-circuit. Au contraire, lorsque la pression d'injection est trop faible, ces sondes indiquent un mauvais remplissage des contacts dès le début du fonctionnement de la génératrice, alors qu'aucun phénomène n'est visible sur les autres courbes. Ensuite, nous observons les mêmes oscillations que sur la courbe de la tension prise entre les bagues de court-circuit.

Les faits majeurs résultant de l'étude expérimentale des instabilités dans les contacts tournants sont:

Les instabilités apparaissent toujours après le maximum de tension et de courant, il en résulte qu'une diminution de la vitesse paraît favoriser les instabilités.

Les mesures de la vitesse et de la dérivée temporelle $\mathrm{du}$ courant indiquent des interruptions du courant.

Les mesures des sondes nous suggèrent un phénomène de vidange et de remplissage successif des contacts liquides.

$\mathrm{La}$ fréquence des oscillations enregistrées est pratiquement constante, $155 \mathrm{~Hz}$, pour de larges variations de la vitesse, du courant et du champ magnétique.

L'amplitude du phénomène croît avec le manque de pression, elle entraîne des dommages mécaniques importants.

3.6.2 Détermination des pressions critiques pour les diverses charges. - Condition expérimentale de stabilité. - Il est remarquable que la limite de la pression entre l'état stable et l'état instable soit aussi nette et que ces phénomènes soient reproductibles. Nous avons vu précédemment que les instabilités se développent au voisinage du courant maximum, c'est-à-dire au voisinage de la vitesse critique. Pour dissocier l'influence de la vitesse et du courant, dans les causes de l'instabilité, nous avons été amenés à chercher expérimentalement les valeurs de la pression critique pour les diverses charges de la génératrice.

Nous avons procédé à une série d'essais à partir des mêmes vitesses initiales en décroissant peu à peu les pressions d'injection du mercure jusqu'à observer le développement des instabilités. Dans un plan de coordonnées $j_{m}^{2}, p$ les points représentatifs du bon fonctionnement des balais liquides et les points représentatifs du fonctionnement avec prise d'instabilités, sont répartis dans deux demi-plans délimités par une droite bien définie. Cette droite représente la variation de la pression critique en fonction du courant maximum obtenu. Nous avons représenté sur la figure 10, la variation de la pression critique en fonction du carré du courant maximum pour les trois séries d'essais effectués en court-circuit, avec la charge purement résistive et avec la bobine spéciale à champ fort.
Les trois droites obtenues sont concourantes pour un courant de $5 \times 10^{5} \mathrm{~A}$ et une pression de $30 \mathrm{kgf} / \mathrm{cm}^{2}$. En dessous de cette valeur du courant, nous n'avons pas observé de développement d'instabilités. La position relative de ces droites montre qu'un accroissement du temps de décharge ou une diminution de la vitesse de rotation du rotor favorise le développement des instabilités.

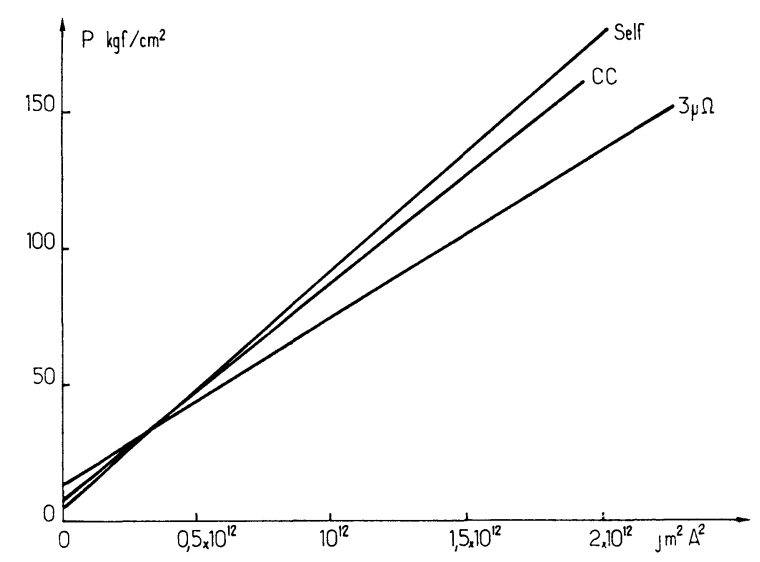

FIG. 10. Evolution de la pression critique.

La condition de stabilité des contacts liquides peut s'exprimer par la relation $p \geqslant p_{c}$.

La variation déterminée expérimentalement de la pression critique $p_{c}$ en fonction de $j_{m}^{2}$ est linéaire, elle est représentée graphiquement sur la figure 10 .

4. Conclusion. - L'expérimentation de la dynamo unipolaire impulsionnelle de $5 \mathrm{MJ}$, conçue et réalisée au Laboratoire de Physique des Plasmas de la Faculté des Sciences d'Orsay a confirmé les prévisions théoriques. Les résultats que nous rapportons dans cet article montrent que ce type de machine auto-excitée et ne comportant pas de matériaux ferromagnétiques permet d'obtenir de hauts niveaux d'énergie sous la forme d'une impulsion courte de courant de forte intensité : plus de $10^{6} \mathrm{~A}$ pendant $0,1 \mathrm{~s}$.

L'emploi de balais liquides à haute densité de courant, constitués par du mercure injecté sous pression, a permis la commutation continue d'une intensité totale dépassant $1,5 \times 10^{6} \mathrm{~A}$ en présence d'un champ magnétique de l'ordre de $5 \mathrm{~T}$. Cependant, afin d'éviter le développement d'instabilités graves dans l'écoulement du mercure, la pression d'injection doit être supérieure à une pression critique croissant avec le maximum du courant. Celle-ci dépend peu de la charge utilisée, elle atteint 180 bars lorsque le courant est de $1,5 \times 10^{6} \mathrm{~A}$.

Grâce à l'expérience acquise lors de cette étude, nous avons depuis entrepris en collaboration avec la Compagnie Electromécanique, la réalisation d'une dynamo de même type emmagasinant une énergie cinétique de $100 \mathrm{MJ}$, qui est actuellement en début d'expérimentation. 


\section{Bibliographie}

[1] Rioux C., Recherche critique d'une forme d'électrotechnique adaptée aux grandes puissances. Thèse de Doctorat ès sciences.

[2] Rioux C., Etude et réalisation d'une dynamo unipolaire à régime impulsionnel ne comportant pas de matériaux ferro-magnétiques. Thèse de Doctorat ès sciences. Masson, Paris 1964.

[3] Lucidarme J., Etude expérimentale d'un écoulement MHD entre deux cylindres coaxiaux. Thèse de Docteur-Ingénieur, Paris 1966.

[4] Hartmann H. et Lazarus F., Experimental investigation on the flow of mercury in a homogenous magnetic field. Det. Kgl. Danske Vidensk. Selskab. Mat fys medd. 15, nos 6 et 7 (1937).

[5] HAHN R., Etude expérimentale des conditions de fonctionnement d'une génératrice unipolaire à régime impulsionnel. Conditions de stabilité des contacts tournants. Thèse de Doctorat $3^{\mathrm{e}}$ cycle, Paris 1967.

[6] Rioux C., Guillet R., Lucidarme J., Projet technique pour une dynamo impulsionnelle accouplée à une bobine à champ intense. Rapport interne au Laboratoire LP 43, nov. 1964. 